Der Kongreß hat mit aller wünschenswerten Deutlichkeit gezeigt, da $\beta$ das Problem der rückständigen Länder weitgehende Beachtung genießt, einmal bei einzelnen Wissenschaftern, die dieses relativ neue Arbeitsfeld mit besonderem Fachinteresse und in der Erwartung betreten, neue Anwendungsbereiche und neue Kontakte für ihre Studien zu finden, zum andern aber auch, weil sich jedermann klar darüber ist, daß die Förderung unterentwickelter Gebiete im Lebensinteresse aller liegt. Offenbar ist in manchen Ländern viel guter Wille, ja Begeisterung zur Mitarbeit vorhanden.

\title{
DAS FLUGZEUG UND DIE ENTWICKLUNG RÜCKSTÄNDIGER GEBIETE
}

\section{ULRICH HALLER}

\author{
Mit 3 Abbildungen
}

Am 16. Dezember 1954 hielt der Gemahl der englischen Königin, der Duke of Edinburgh, vor der Royal Aeronautical Society einen vielbeachteten Vortrag zu diesem Thema. Seine Ausführungen regen an, dieses immer aktueller werdende Problem einmal von einem etwas umfassenderen Standpunkt aus zu würdigen, umsomehr als das Flugwesen den Landschaftscharakter jener Gebiete entscheidend umgestalten dürfte.

\section{DIE ANWENDUNGSBEREICHE DES FLUGZEUGES}

Wir können die nachstehenden Verwendungszwecke unterscheiden, die sich jedoch gegenseitig überschneiden, da die vorhandenen Flugzeuge möglichst universell verwendet werden müssen, um eine angemessene Amortisation zu gewährleisten. Der regelmäßige Passagierverkehr und die Militärluftfahrt wurden nicht berücksichtigt, da diese nicht spezifisch für die besprochenen Gebiete sind.

Düngung. In der Landwirtschaft wird heute das Flugzeug in erster Linie zum Düngen, Säen, zur Schädlingsbekämpfung und zur Überwachung von Feld und Wald verwendet.

Das Düngen aus der Luft ist eine Spezialität von Neuseeland. Dabei wird Dünger aus Flugzeugen über schlecht zugänglichen Gebieten abgeworfen, um die Grasnarbe zu verbessern. Im Auckland District (Neuseeland) bestreute man so etwa 360 ha zweimal mit rund $600 \mathrm{~kg}$ Phosphat- und Kalidünger pro ha. In der Folge konnte der Bestand an Mutterschafen von 1200 auf 2300 erhöht werden. Die Bedeutung der Düngung aus der Luft zeigt sich auch darin, daß 1954 in Neuseeland 162 Flugzeuge dafür verwendet wurden, die mehr als 140000 Tonnen Dünger über $6000 \mathrm{~km}^{2}$ ausstreuten. Die amtlichen Stellen glauben, daß dadurch die Fleischproduktion von Neuseeland innerhalb von zehn Jahren um $50 \%$ gesteigert werden könne.

Schädlingsbekämpfung. Bei der Schädlingsbekämpfung ergeben sich für das Flugzeug Möglichkeiten, wo es den erdgebundenen Bekämpfungsarten weit überlegen ist. So werden beispielsweise in Ostafrika Flugzeuge zur Überwachung und Vernichtung von Heuschreckenschwärmen verwendet. 1954 waren zehn umgebaute Sportflugzeuge in Kenya und Tanganjika gegen eine Invasion von etwa 50000 Millionen Heuschrekken eingesetzt, die sich über eine Fläche von $1300 \mathrm{~km}^{2}$ ausgebreitet hatten. Diese Heuschrecken repräsentierten zusammen ein Gewicht von gegen 100000 Tonnen. Eine große Zahl von Schwärmen konnte vernichtet werden, doch kamen schließlich die wenigen Flugzeuge gegen die Heuschreckenfluten nicht mehr auf. Während des Kampfes wurden mit einem Liter Gift etwa 100000 Heuschrecken getötet, während mit jedem ausgegebenen Pfund Sterling Pflanzungen im Wert von etwa \& 110 gerettet werden konnten.

Ebenfalls vorwiegend in Afrika wird das Flugzeug gegen das wohl größte Entwicklungshindernis des Kontinentes, gegen die Tse-tse-Fliege, eingesetzt. Allein in Tanganjika sind heute noch $75 \%$ des Landes durch dieses Insekt verseucht und in der Folge für den Menschen unbewohnbar. Alle diese Gebiete ließen sich aus der Luft 


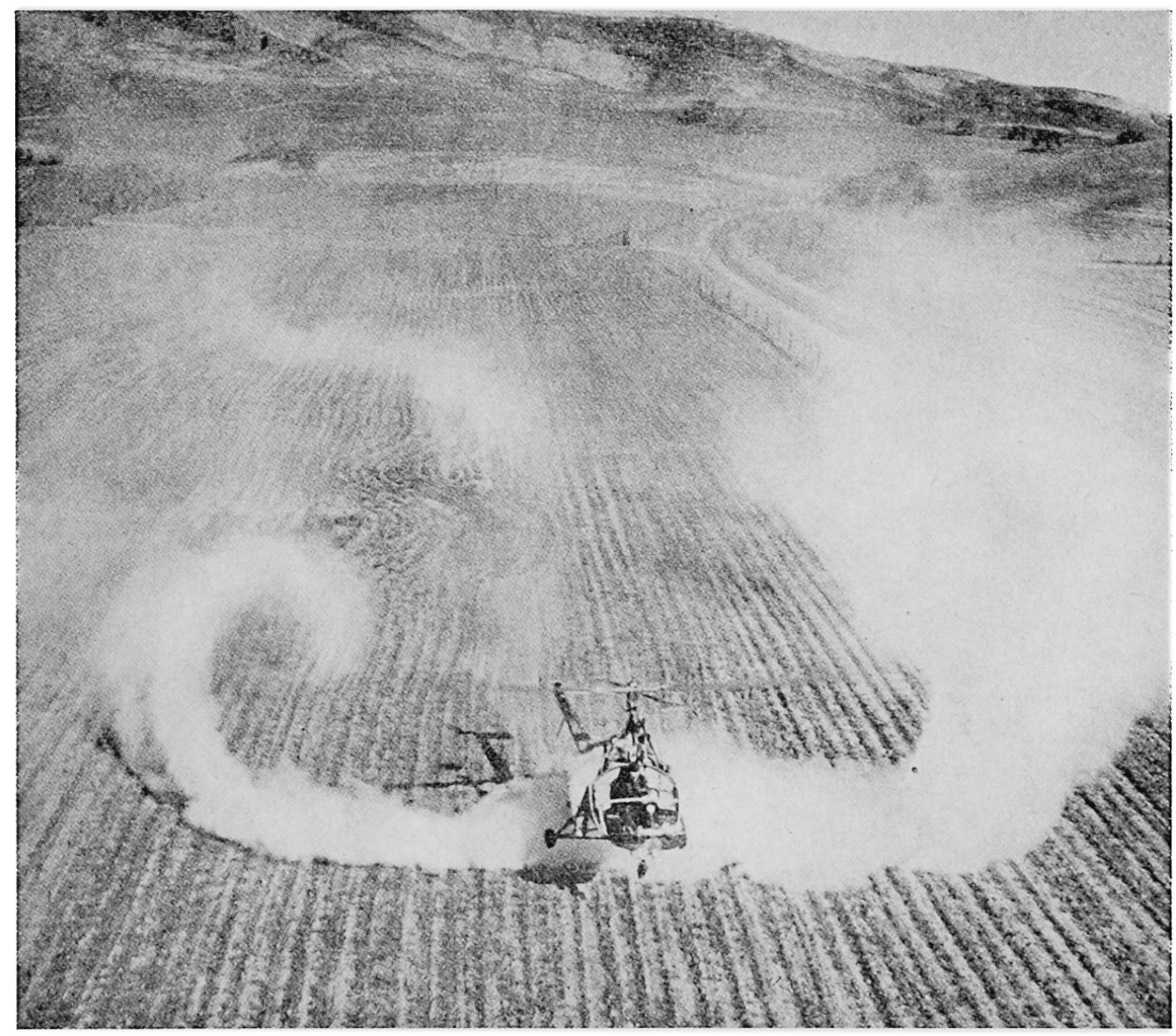

Schädlingsbekämpfung durch Helikopter in Kalifornien. Durch den abwärts gerichteten Luftstrom der Rotorblätter werden die Chemikalien gleichmäßig über die Pflanzen verteilt. Nach Esso Air World

säubern. Doch wäre eine dreimalige Behandlung nötig, was vorläufig noch eine zu große Ausgabe darstellt.

Die Schädlings- und Seuchenbekämpfung aus der Luft hat neuerdings weltweite Bedeutung erlangt. So verwendete man beispielsweise auch im Unterwallis Flugzeuge zum Bespritzen der Obstkulturen. Die große Entwicklung der verwendeten Giftstoffe brachte es mit sich, daß heute nur noch etwa 50 Liter pro ha benötigt werden, während vor wenigen Jahren noch die zehnfache Menge erforderlich war. Die Tatsache, daß die Chemikalien schon in kleinen Dosierungen wirksam sind, ist in erster Linie verantwortlich für die immer stärker werdende Verwendung des Flugzeuges in den letzten zwölf Jahren. Seine Vorzüge gegenüber den erdgebundenen Mitteln liegen vor allem darin, daß das Flugzeug fast unabhängig von der Beschaffenheit des zu behandelnden Gebietes operieren kann. Es vermag dicht stehende Kulturen, Baumgipfel, Wüsten und Sümpfe zu erreichen und dies mit einer Geschwindigkeit, die anderweitig nicht möglich wäre.

Vermessung und Überwachung. Es ist bekannt, daß seit Jahren große und schwer zugängliche Gebiete der Erde mittelst des Flugzeugs luftphotogrammetrisch, vermessen werden. Dies gilt insbesondere von den riesigen Arealen Canadas, Australiens, Afrikas und der Polargebiete, wo die Vermessung vor allem zur Prospektion, zum Bahnbau usw. nötig ist. In Südafrika zum Beispiel wurden $80000 \mathrm{~km}^{2}$ vermessen, um die 


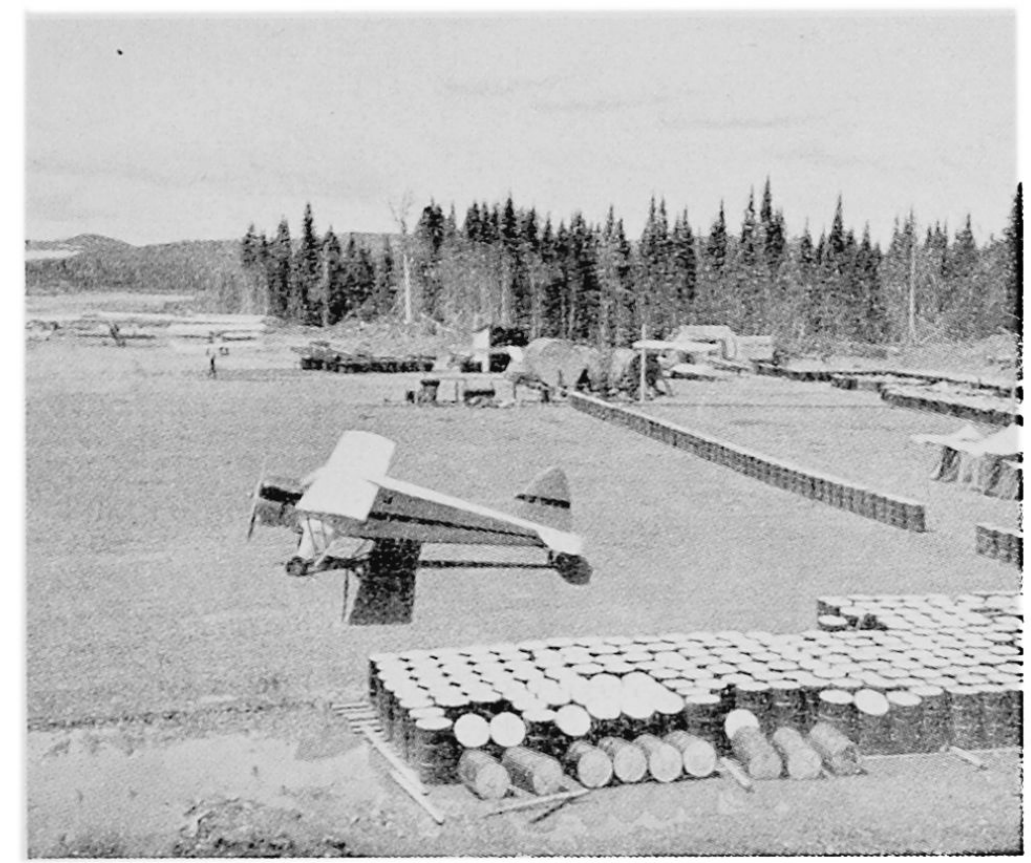

„Budworm City“, die mitten aus dem „Urwald“ von New Brunswick herausgehauene Basis der zwanzig Flugzeuge, die im Juni 1952 auf einer Fläche von $800 \mathrm{~km}^{2}$ gegen Baumschädlinge eingesetzt wurden.

Nach Esso Air World

beste Linienführung für eine neue Bahnstrecke zu finden. An der Goldküste kartierte man mit Flugzeugen $40000 \mathrm{~km}^{2}$ für ein geplantes Kraftwerksystem, während in der Indischen Union und in Pakistan für geologische und Irrigationszwecke 450000 resp. $800000 \mathrm{~km}^{2}$ aus der Luft kartiert wurden. Zur Prospektion Nord-Canadas, Alaskas und Australiens sind zahlreiche neue Methoden entwickelt worden. Neben der herkömmlichen Luftbildinterpretation helfen Messungen des Erd- und Elektromagnetismus sowie der Radioaktivität, die gleichzeitig im Fluge vorgenommen werden können, neue Minerallager aufzuspüren.

Außerdem findet das Flugzeug Verwendung zur Überwachung der Erosion in anderweitig schlecht zugänglichen Gegenden, während in Canada die Forstpläne mit Hilfe von Luftbildern aufgestellt werden. Canada verfügt im weiteren über eine systematische Luftüberwachung seiner Wälder als Schutz gegen Brände.

Der Vorteil des Flugzeuges liegt in allen diesen Fällen darin, daß die benötigte Zeit äußerst gering ist. Beim Bau des Kitimat-Kraftwerkes in West-Canada war die beste Linienführung für eine $80 \mathrm{~km}$ lange Kraftleitung durch ein gegen $2000 \mathrm{~m}$ hohes Bergland zu suchen. Mit Hilfe von Helikoptern wurde diese Arbeit innerhalb von 30 Flugstunden erledigt, während für jede andere Methode mindestens 3 Jahre benötigt worden wären. Ein weiteres Beispiel: Die Kontrolle des Frazer River Canyons in British Columbia, die bisher zu Fuß fünf Jahre gedauert hatte, kann heute mit dem Flugzeug in einer Saison vorgenommen werden.

Luftfrachten. In Europa gilt die Luftfracht in der Regel als teuer und vielfach als unwirtschaftlich, da für die meistens relativ kurzen Distanzen billigere Transportmittel zur Verfügung stehen. Anders verlief die Entwicklung in weiträumigen und schwach besiedelten Gegenden wie beispielsweise in Canada, wo schon $193711000 \mathrm{t}$ mit dem Flugzeug befördert worden waren im Vergleich zu $4500 \mathrm{t}$ in der gleichen Zeitspanne in den USA. Die Anfänge der Luftfracht gehen in Canada schon in die Zwanzigerjahre zurück. Jede weitere Entwicklung des Nordens ist zwangsläufig vom Flugzeug abhängig.

Wählen wir als Beispiel die Eldorado Uranminen in Port Radium am Großen Bärensee, wenige Kilometer südlich des Polarkreises. Abgesehen von besonders großen und unhandlichen Maschinenteilen erfolgt die Versorgung dieser Minenstadt ausschließlich auf dem Luftwege vom gegen $2000 \mathrm{~km}$ entfernten Edmonton aus. Mit Hilfe von zwei zweimotorigen Transportern werden durchschnittlich im Jahr 3000 Passagiere und 3000 t Fracht befördert. Die einzige andere Transportmöglichkeit ist der Wasserweg, der jedoch nur während eines Monats im Jahr benützt werden 


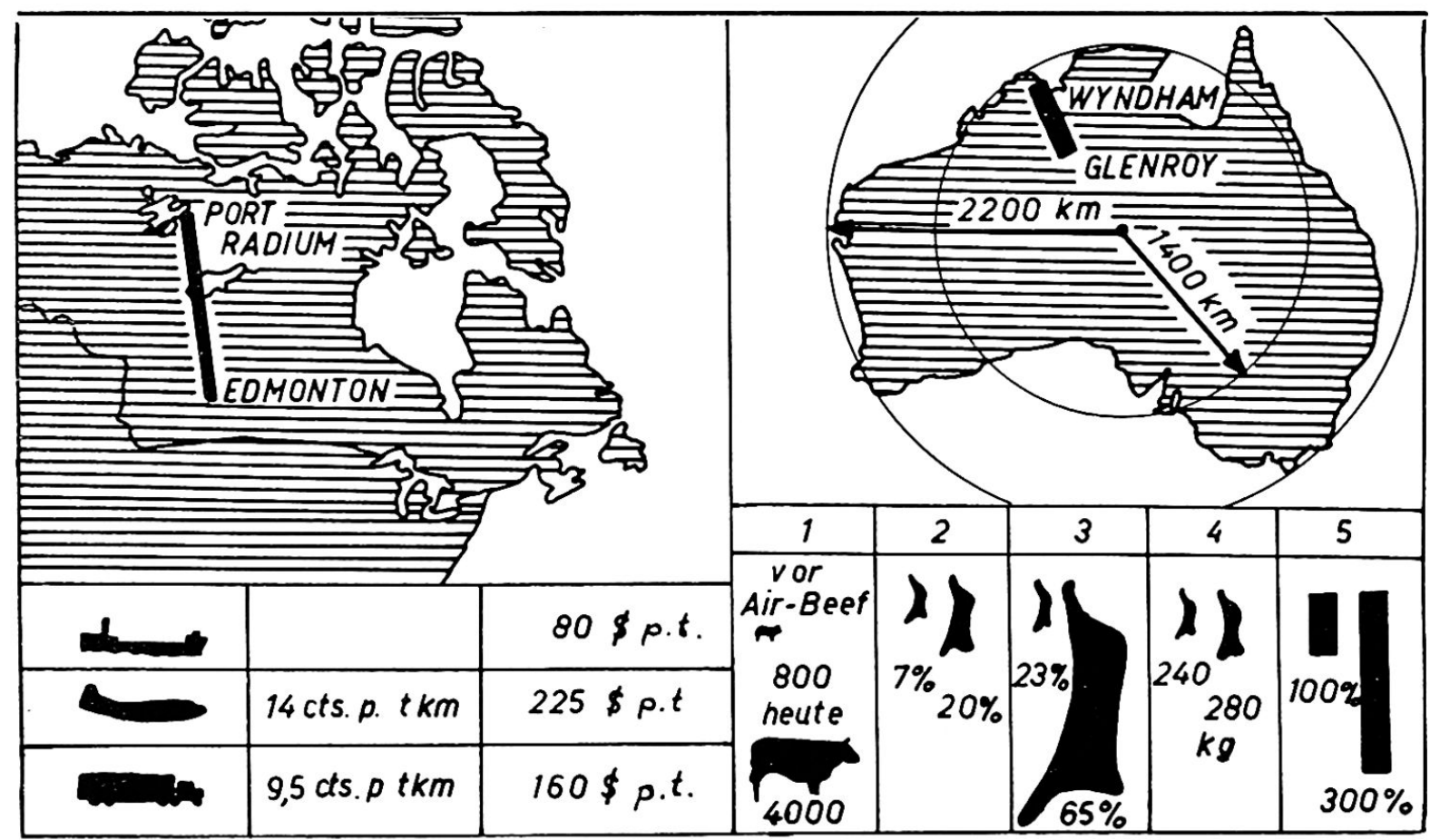

Beispiele der "Outback Aviation“. Links: Versorgung von Port Radium. Gegenüberstellung der Kosten pro Tonnenkilometer und pro Tonne für Schiff-, Luft- und Straßentransport. Rechts : Experiment "Air-Beef“ 1 jährlich geschlachtete Stück Vieh, 2 zur Verarbeitung gelangtes Vieh in $\%$ von 1, 3 Anteile der Exportqualität am produzierten Fleisch, 4 mittleres Gewicht pro Stück Vieh, 5 Gewinnvergrößerung durch "Air-Beef".

Nach Flight 1954

kann, so daß heute praktisch nur die Produkte der Minen verschifft werden. Die Kosten pro Tonne stellen sich bei der Schiffahrt auf 80 \$, beim Lufttransport auf $225 \$$. Der (hypothetische) Straßentransport würde nach den gültigen Normen auf etwa $160 \$$ zu stehen kommen. Die relativ geringen Kosten des Lufttransportes ergeben sich daraus, daß der Ausnützungsgrad der Flugzeuge fast $100 \%$ erreicht (Swissair 55-60\%). Durch das Flugzeug stehen diese weit abgelegenen Vorposten in dauernder Verbindung mit den übrigen Landesteilen; frisches Gemüse und Obst, Zeitungen usw. sichern den Bewohnern ein normales, volles Leben.

Einen weitern Beweis für die Nützlichkeit des Flugzeuges für die Entwicklung weit abgelegener Gebiete liefert das sog. „Air-Beef“-Experiment von Glenroy in Nordwest-Australien. Vor der Einführung des Flugzeuges hatten die Viehherden die Distanz von $500 \mathrm{~km}$ bis zum nächsten Hafen (Wyndham) zu Fuß zurückzulegen, wobei schließlich noch $7 \%$ der Tiere die Konservenfabriken erreichten. Um diese großen Verluste zu vermeiden, wurde versuchsweise in Glenroy ein Schlachthaus errichtet, von wo aus das gefrorene Fleisch mit dem Flugzeug in die Konservenfabriken der Häfen transportiert wird.

Damit haben sich für Australien ungeahnte Möglichkeiten eröffnet. Man glaubt, daß mit der neuen Methode - Schlachthäuser im Inland, Lufttransport nach den Häfen - die Konservenproduktion innerhalb von zehn Jahren auf das Doppelte gesteigert werden kann.

Schließlich sei noch auf die Bahnlinie vom franco-canadischen Fischerdorf Seven Islands am St. Lawrence nach dem $600 \mathrm{~km}$ nordwärts gelegenen Minenort Burnt Creek hingewiesen, die beinahe vollständig mit Hilfe des Flugzeuges erbaut worden ist. Um die Bauzeit abzukürzen, wurde die Bahnlinie gleichzeitig von vier Punkten aus vorgetrieben, die auf dem Luftwege mit dem erforderlichen Material und der Verpflegung versorgt wurden.

Ärztlicher Hilfs- und Rettungsdienst. Wohl die bekanntesten Beispiele dafür sind der sog. "Flying Doctor Service» in Australien sowie die Rettungsflugwacht in der Schweiz. Auch in Canada, Schweden und Dänemark u. a. a. O. existieren entsprechende Organisationen. Die Möglichkeit, erkrankte und verunfallte Personen schnellstens aus dem Dschungel in die Spitäler zu bringen, hat beispielsweise auch die Shell Oil Co. dazu bewogen, in Borneo neben den Kurierflugzeugen zwischen den Ölfeldern Hilfsflugzeuge einzusetzen. Damit konnte kürzlich ein an Typhus erkrankter Angestellter fünf Stunden nach Einlaufen der Funkmeldung ins Spital eingeliefert werden, während die Reise zu Lande gegen 40 Stunden in Anspruch genommen hätte. 


\section{Zusammenfassung}

Die Grundkonzeption des Flugwesens in unseren Gegenden ist gänzlich verschieden von derjenigen der erwähnten Länder. Während das Fliegen bei uns trotz seiner großen volkswirtschaftlichen Bedeutung im gesamten gesehen auch heute noch das Vorrecht einer Minderheit darstellt, ist dort das Flugzeug ein lebenswichtiger Teil der Zivilisation geworden. Es nimmt im Leben der Bevölkerung jene Stelle ein, die bei uns durch die Eisenbahn und die übrigen öffentlichen Verkehrsmittel belegt wird. In den meisten Fällen wird das Flugzeug nicht darum verwendet, weil es billiger oder schneller ist, sondern weil überhaupt keine andere Verkehrsmöglichkeit besteht. Es kann daher ruhig gesagt werden, daß auch die zukünftige Entwicklung von großen Teilen der von der heutigen Zivilisation noch nicht oder nur wenig berührten Länder zur Hauptsache auf der Luftfahrt beruhen wird und daß davon auch wesentliche Umgestaltungen der Landschaft zu erwarten sind.

\section{LITERATUR}

„Outback aviation“. Flight and aircraft engineer. 66, 1954, 922-25. - Illingworth, F.: „Labradors iron-ore airlift.“ Flight. 59, 1951, 690-91. - KeITH, R. A.: „The Flying Firemen.“ Esso Air World. 4, 1951, 69-72. - „Air Beef.“ Esso A. W. 3, 1950, 24-25. - „Pioneering in British Guyana.“ Esso A. W. 2, 1949, 19-21. - „Air Supply within the Arctic Cycle." Esso A.W. 2, 1949. $128-30$. - "The Australian Flying Doctor.“ Esso A. W. 2, 1949, 163-165. - Bridgman, J. M.: „The Airborne Magnetometer.“ Esso A.W. 2, 1949, 73-76.

\section{DIE ARBEITSTAGUNG DES VEREINS SCHWEIZERISCHER GEOGRAPHIELEHRER VOM 15. JANUAR 1955}

\section{Kurt Hintermann und Ernst WinkLer}

Einer guten Eingebung folgend rief der Vorstand des Vereins schweizerischer Geographielehrer seine Mitglieder auf den 15. Januar 1955 nach Olten zu einer Arbeitstagung zusammen. Absicht und Ziel war, die Schulgeographen wieder einmal ins $\mathrm{Ge}$ spräch über aktuelle Fragen des Unterrichts zu bringen, um daraus gegebenenfalls Richtlinien für die weitere Tätigkeit zu gewinnen.

Der Einladung folgten 55 Kollegen der Schul- und Hochschulgeographie und verwandter Fächer - eine recht ansehnliche Zahl, obwohl damit nur ein guter Viertel der Vereinsmitglieder ihr Interesse an einer Aussprache bekundete. Der Präsident, Prof. OTto Wernil, Aarau, auf den die Anregung zur Tagung in wesentlichen zurückgeht, begrüßte die Teilnehmer im hellen Singsaal des Froheimschulhauses und gab zunächst eine knappe Programmvorschau. Sie sah drei Haupttraktanden vor: Referate und Diskussion, Ausstellung und eine Geschäftssitzung. Die beiden letztern wurden durch die Haupttraktanden: Vorträge mit Diskussion eingerahmt, die man unter drei Hauptthemata zusammengefaßt hatte.

Als erstes kam «Die Behandlung einer Landschaft in der Mittelschule 》zur Erörterung. Dr. Werner Nigg, Lehrer für Geographie an der Kantonalen Handelsschule Zürich, umriß in bemerkenswerter Kürze und Klarheit, das Beispiel der Bündner Herrschaft wählend, wesentliche Momente dieses wohl zentralsten Gegenstandes der Schulgeographie nicht nur, sondern der Geographie überhaupt. Er wies vor allem auf den offensichtlichen Kontrast zwischen dem sogenannten «länderkundlichen Schema » und einer mehr die «Landschaftsdominanten 》 herausarbeitenden Methode hin. Seine Ausführungen gipfelten in grundsätzlichen Fragen nach der Wünschbarkeit der Landschaftsdiskussion und nach der Definition des Begriffes Landschaft in der (Mittel-) Schule, nach der Verwendbarkeit von «Schema » und «Dominantenlehre » und andern Behandlungsmöglichkeiten und schließlich nach dem Problem der Synthese im landschaftskundlichen Unterricht. Die hieran anknüpfende Diskussion brachte zum Ausdruck, daß Mittel- und Volksschule eines länderkundlichen «Schemas » kaum ent- 